\title{
FISH mapping of two putative keratin gene clusters on cat (Felis catus) chromosomes
}

\section{Other Conference Item}

\section{Author(s):}

Szczerbal, I.; Switonski, M.; Michalak, E.; Rogalska-Niznik, N.; Szamalek, J.; Schelling C.

Publication date:

2003

Permanent link:

https://doi.org/10.3929/ethz-b-000056388

Rights / license:

In Copyright - Non-Commercial Use Permitted

Originally published in:

Journal of Heredity 94(1), https://doi.org/10.1093/jhered/esg011 


\title{
FISH Mapping of Two Putative Keratin Gene Clusters on Cat (Felis catus) Chromosomes
}

\author{
I. Szczerbal, M. Switonski, E. Michalak, N. Rogalska-Niznik, J. Szamalek, and C. Schelling \\ From the Department of Genetics and Animal Breeding, August Cieszkowski Agricultural University, Poznán, Poland \\ (Szczerbal, Switonski, Michalak, Rogalska-Niznik, and Szamalek) and the Department of Animal Sciences, Swiss Federal \\ Institute of Technology and Faculty of Veterinary Medicine, University of Zurich, Zurich, Switzerland (Schelling). This study was \\ supported by the Foundation for Polish Science (contract no. 13/2000). This paper was delivered at the Advances in Canine \\ and Feline Genomics symposium, St. Louis, MO, May 16-19, 2002.
}

Address correspondence to Marek Switonski, Department of Genetics and Animal Breeding, August Cieszkowski Agricultural University of Poznán, Wolynska 33, 60-637 Poznán, Poland, or e-mail: switonsk@jay.au.poznan.pl.

\begin{abstract}
Genes encoding keratins are evolutionary highly conserved and clustered in two linkage groups in mammalian genomes. Canine keratin $9(\mathrm{~K}-9)$ and keratin $2 \mathrm{e}(\mathrm{K}-2)$ cosmid-derived gene probes were used to localize the acidic and basic-neutral keratin gene clusters to cat chromosomes E1q12 and B4q15, respectively. The status of the physical map of the cat genome is discussed.
\end{abstract}

Although an integrated feline radiation and genetic linkage map comprised of 600 markers has been recently published (Sun et al. 2001) and a comparative genome map for dogs, cats, and humans exists (Yang et al. 2000), very few genes, such as MYC (Cho et al. 1997), IGL (Cho et al. 1998), TCRB (Lee and Cho 1999), TNFSF6 (Fujino et al. 2001b), and TNFRSF6 (Fujino et al. 2001a), have been mapped by fluorescence in situ hybridization (FISH) onto cat chromosomes.

Keratin genes are highly conserved across mammalian genera. These genes encode the epithelial keratins, which are differentially expressed in the various types of epithelia and the hair keratins, and are involved in the formation of hard keratinized structures. The keratins can be divided into two classes: acidic type I keratins and basic-neutral type II keratins, which form the intermediate filament network of the cytoskeleton (Moll et al. 1982). Genes encoding keratins have been found to be clustered in two linkage groups that map to two different chromosomes in mammalian species (human, mouse), including the dog (Keller et al. 1998; Miller et al. 1999). Mutations in human keratin genes are quite frequent and can cause inherited skin disorders (IshidaYamamoto et al. 1998). There are also some reports of such diseases in dogs (Mecklenburg et al. 2000). Thus development of knowledge of feline and canine keratin genes seems to be important. In this article fluorescence in situ hybridization (FISH) mapping of two keratin gene clusters in the cat genome is described.

\section{Materials and Methods}

Chromosome preparations were obtained from short-term lymphocyte cultures. Identification of the chromosomes was performed by the Q-banding technique prior to FISH. International nomenclature for cat chromosomes (Ford et al. 1980) and data originating from comparative chromosome painting (Yang et al. 2000) were followed.

FISH was performed with the use of two canine-derived cosmid clones containing keratin genes described as $K-2$ (keratin 2e; basic) and $K-9$ (keratin 9; acidic). The clones were labeled with biotin-16-dUTP by the random priming method and then the standard protocol for FISH was applied (Keller et al. 1998). Slides were analyzed under a fluorescent microscope (Nikon E 600 Eclipse) equipped with a CCD camera driven by computer-aided software (Lucia).

\section{Results and Discussion}

The putative acidic and basic-neutral keratin gene clusters were physically assigned to cat chromosomes E1q12 and B4q15, respectively (Figures 1 and 2). On the E1 chromosome, the nucleolar organizer region was mapped by FISH (Szczerbal et al., unpublished data), while on the B4 chromosome, no other loci have been physically localized. Our results confirm data from a comparative human, canine, and feline genome map that was developed by reciprocal 

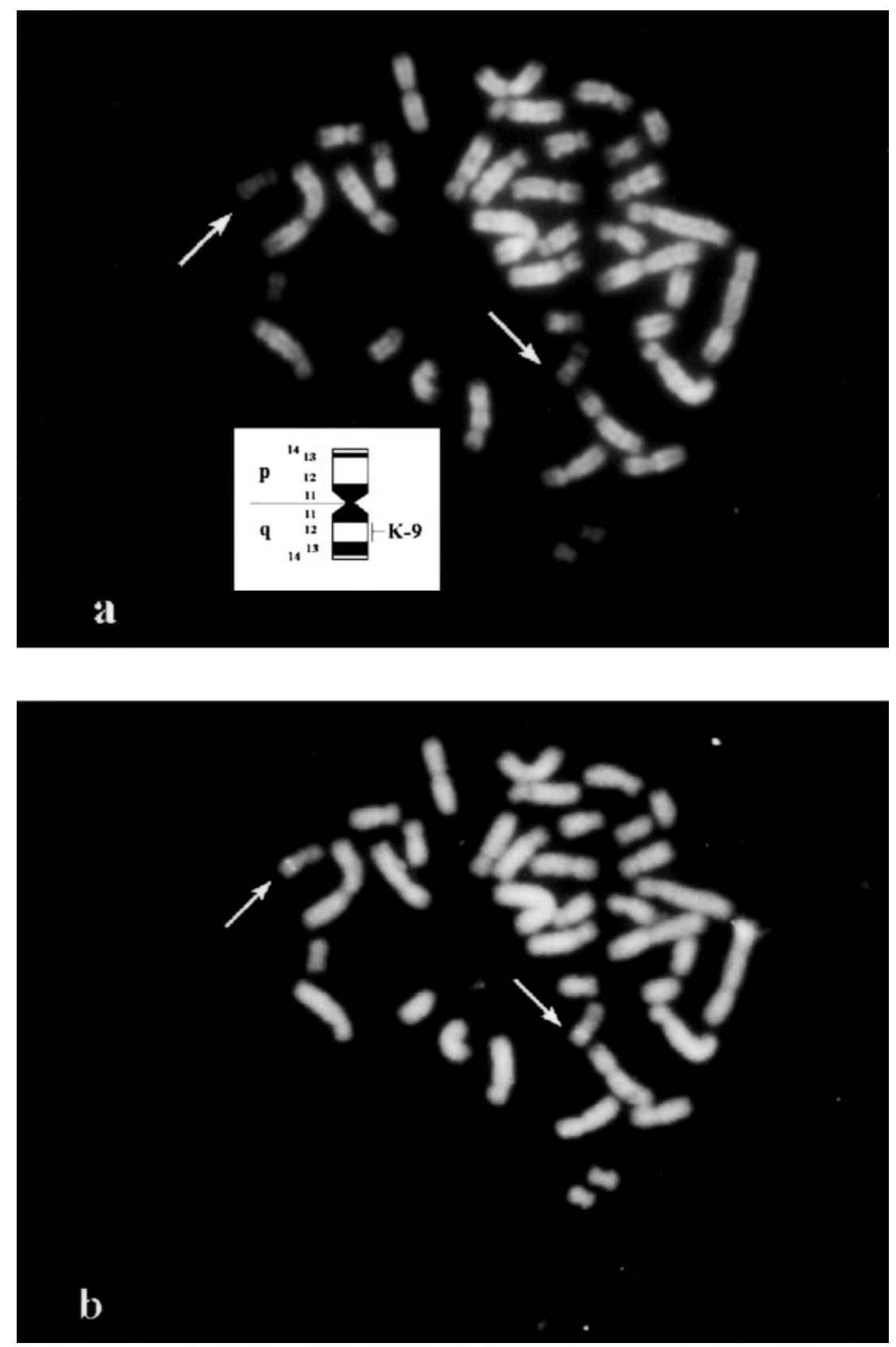

Figure I. Localization of the K-9 probe to the cat chromosome E1: (a) Q-banding and an ideogram of the E1 chromosome; (b) FISH.

chromosome painting (Yang et al. 2000). According to that study, feline chromosome E1q is homologous to human chromosome 17 and canine chromosomes 9 and 5. It is known that the human and canine acidic keratin gene clusters have been mapped to HSA 17 (Rosenberg et al. 1988) and CFA 9 (Miller et al. 1999), respectively. The feline B4q chromosome is homologous to human chromosome 12 and fragments of canine chromosomes 2, 10, 15, and 29 (Yang et al. 2000). Human and canine basic-neutral keratin gene clusters have been mapped to HSA 12 (Popescu et al. 1989) and CFA 27 (Miller et al. 1999), respectively.
While the chromosomal assignment of the acidic keratin gene cluster to cat chromosome E1 fully confirms the results of the comparative genome map (dog, human, and cat) and the physical assignments in human and dog, there are inconsistencies in the results for the basic-neutral keratin gene cluster in these three species. Miller et al. (1999) mapped the putative canine basic-neutral keratin cluster to CFA 27, the data from Yang et al. (2000) would predict a localization of the same locus on CFA 29. The discrepancy can be explained by the different chromosome numbering systems used by these two groups. Graphodatsky et al. (2000) 

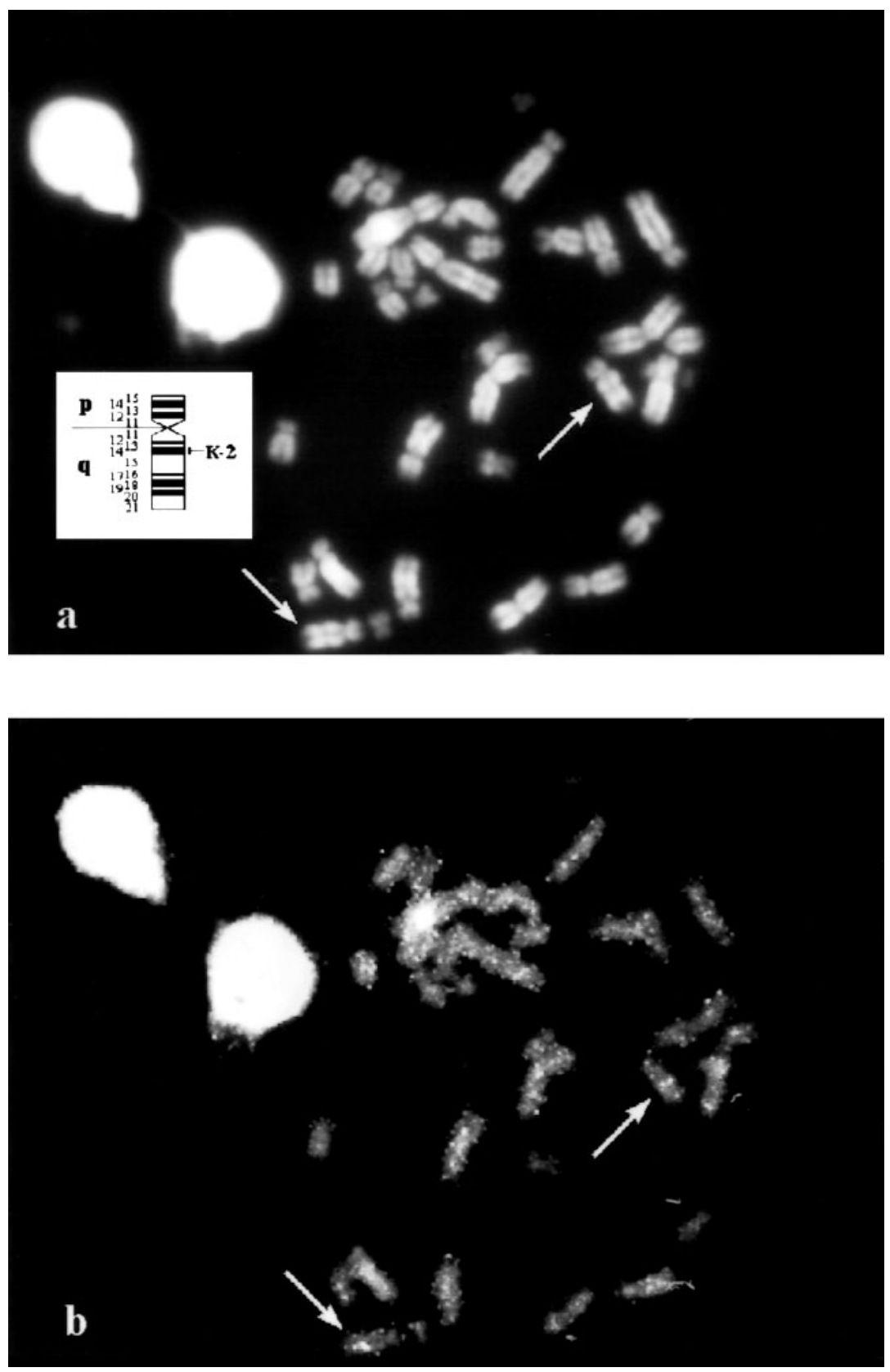

Figure 2. Localization of the $K-2$ probe to the cat chromosome B4: (a) Q-banding and an ideogram of the B4 chromosome; (b) FISH.

showed that the canine chromosome CFA 27, identified by Miller et al. (1999), corresponds to the CFA 29 described by Yang et al. (2000). Therefore, it can be concluded that physical assignments of the two keratin gene clusters in dogs, cats, and humans are in accordance with comparative genome maps of these three species. Comparative analysis of chromosome localization of the keratin gene clusters in some species of the family Canidae was performed by
Szamalek et al. (2002). These data also indicate that the basic keratin gene cluster resides on canine chromosome CFA29.

The results of this article contribute to the further development of the domestic cat genome map and will be useful in clarification of diseases (e.g., skin) in which keratin genes might be involved. In addition, we clearly demonstrate that FISH remains an important tool for enhancing the use of comparative genome maps. 


\section{References}

Cho KW, Satoh H, Youn HY, Watari T, Tsujimoto H, and Hasegawa A, 1997. Assignment of the feline c-myc gene (MYC) to cat chromosome F2q21.2 by fluorescence in situ hybridisation. Cytogenet Cell Genet 78:135-136.

Cho KW, Tsujimoto H, Hasegawa A, and Satoh H, 1998. The cat immunoglobulin lambda light chain gene maps to chromosome D3p12p11. Mamm Genome 9:178-179.

Ford CE, Pollock DL, and Gustavsson I, 1980. Proceedings of the First International Conference for the Standardisation of Banded Karyotypes of Domestic Animals. Hereditas 92:145-162.

Fujino Y, Mizuno T, Masuda K, Ohno K, Satoh H, and Tsujimoto H, 2001a. Assignment of the feline Fas ligand gene (TNFRSF6) to chromosome D2p13-p12.2 by fluorescence in situ hybridisation. Cytogenet Cell Genet 95:122-124.

Fujino Y, Mizuno T, Masuda K, Ohno K, Satoh H, and Tsujimoto H, 2001b. Assignment of the feline Fas ligand gene (TNFSF6) to chromosome F1q12q13 by fluorescence in situ hybridisation. Cytogenet Cell Genet 94:92-93.

Graphodatsky AS, Yang F, O’Brien PCM, Serdukova N, Milne BS, Trifonov V, and Ferguson-Smith MA, 2000. A comparative chromosome map of the arctic fox, red fox and dog defined by chromosome painting and high resolution G-banding. Chromosome Res 8:253-263.

Ishida-Yamamoto A, Tanaka $\mathrm{H}$, Nakane $\mathrm{H}$, Takahashi $\mathrm{H}$, and Iizuka $\mathrm{H}$, 1998. Inherited disorders of epidermal keratinization. J Dermatol Sci 18 139-154.

Keller RC, Switonski M, Jörg H, Ladon D, Arnold S, and Schelling C, 1998. Chromosomal assignments of two putative canine keratin gene clusters. Anim Genet 29:141-143.

Lee JH and Cho KW, 1999. Assignment of TCRB encoding the T-cell receptor beta chain to cat chromosome A2q25-q26 by fluorescence in situ hybridisation. Cytogenet Cell Genet 84:109-110.

Mecklenburg L, Hetzel U, and Ueberschar S, 2000. Epidermolytic ichthyosis in a dog: clinical, histopathological, immunohistochemical and ultastructural findings. J Comp Pathol 122:307-311.

Miller AB, Breen M, and Murphy KE, 1999. Chromosomal localization of acidic and basic keratin genes of the domestic dog. Mamm Genome 10:371-375

Moll R, Franke WW, Schiller DL, Geiger B, and Krepler R, 1982. The catalogue of human cytokeratins: patterns of expression in normal epithelia, tumours and cultured cell. Cell 31:11-24.

Popescu NC, Bowden PE, and DiPaolo JA, 1989. Two type II keratin genes are localized on human chromosome 12. Hum Genet 82:109-112.

Rosenberg M, RayChaudhury A, Shows TB, Le Beau MM, and Fuchs E, 1988. A group of type I keratin genes on human chromosome 17: characterization and expression. Mol Cell Biol 8:722-736.

Sun S, Murphy WJ, Menotti-Raymond M, and O'Brien S, 2001. Integration of the feline radiation hybrid and linkage maps. Mamm Genome 12: 436-441.

Szamalek J, Szczerbal I, Rogalska-Niznik N, Switonski M, Ladon D, and Schelling C, 2002. Chromosomal localization of two keratin gene families in the karotype of three species of the family Canidae. Anim Genet 33: 404-405.

Yang F, Graphodatsky AS, O’Brien PCM, Colabella A, Solanky N, Squire M, Sargan DR, and Ferguson-Smith MA, 2000. Reciprocal chromosome painting illuminates the history of genome evolution of the domestic cat, dog and human. Chromosome Res 8:393-404.

\section{Corresponding Editor: William Murphy}

